\title{
Criminologie
}

\section{Dangerosité, risque et technologies du pouvoir}

\section{John Pratt}

Volume 34, numéro 1, printemps 2001

La notion de risque dans la gestion pénale

URI : https://id.erudit.org/iderudit/004756ar

DOI : https://doi.org/10.7202/004756ar

Aller au sommaire du numéro

Éditeur(s)

Les Presses de l'Université de Montréal

ISSN

0316-0041 (imprimé)

1492-1367 (numérique)

Découvrir la revue

Citer cet article

Pratt, J. (2001). Dangerosité, risque et technologies du pouvoir. Criminologie, 34(1), 101-121. https://doi.org/10.7202/004756ar

\section{Résumé de l'article}

Dans cet article, nous étudierons la signification de la législation relative aux délinquants dangereux et certaines des questions théoriques qui en découlent. La discussion portera sur l'évolution de la " dangerosité » en tant que construction sociale au cours de l'histoire et, dans le même courant, sur les modifications de son mode d'étalonnage. Enfin, nous verrons comment la législation moderne sur la dangerosité implique l'utilisation d'une stratégie de contrôle, «l'actuarialisme », passée largement inaperçue ; en fait, cette stratégie est plus susceptible d'avoir un effet sur le comportement des victimes potentielles que sur les délinquants dangereux eux-mêmes. 


\title{
Dangerosité, risque et technologies du pouvoir
}

\author{
John Pratt \\ Reader in Criminology \\ Institute of Criminology \\ University of Wellington • New Zealand \\ john.pratt@vuw.ac.nz
}

RÉSUmÉ - Dans cet article, nous étudierons la signification de la législation relative aux délinquants dangereux et certaines des questions théoriques qui en découlent. La discussion portera sur l'évolution de la « dangerosité » en tant que construction sociale au cours de l'histoire et, dans le même courant, sur les modifications de son mode d'étalonnage. Enfin, nous verrons comment la législation moderne sur la dangerosité implique l'utilisation d'une stratégie de contrôle, « l'actuarialisme », passée largement inaperçue ; en fait, cette stratégie est plus susceptible d'avoir un effet sur le comportement des victimes potentielles que sur les délinquants dangereux eux-mêmes.

ABSTRACT - This paper examines the significance of dangerous offender legislation and some of the theoretical issues that this raises. This involves discussion of the way in which "dangerousness" as a social construct has changed historically and similarly the mode of its calibration. Ultimately, the dangerousness legislation today involves the use of a largely unnoticed strategy of control - actuarialism - and seems more likely to have an effect on the behaviour of potential victims of crime rather than dangerous offenders themselves. 


\section{Dangerosité, risque et technologies du pouvoir}

À l'heure actuelle, la « dangerosité » semble être l'un des thèmes les plus courants dans les juridictions de droit criminel des pays anglophones ${ }^{1}$. Il existe une gamme de législations comportant des mesures qui permettent aux tribunaux de condamner les individus jugés « délinquants dangereux » à des périodes d'emprisonnement indéfinies, habituellement qualifiées de détention préventive, tout en imposant souvent des restrictions spécifiques relativement à leur libération conditionnelle ultérieure. Cette situation se traduit souvent par une législation sur les prédateurs sexuels, les three strikes laws aux Etats-Unis, ou leur équivalent ailleurs ; il s'agit, succinctement, de la réactivation de lois plus anciennes contre les multirécidivistes.

Dans cet article, je considérerai la signification sociologique de ces mesures, et les questions qui en découlent concernant le concept même de danger, la gestion du risque de crime, et les modalités de contrôle social dans la société moderne. Cette analyse ne conteste pas le fait que certains criminels soient dangereux. Ce que je tente d'établir, toutefois, c'est que la dangerosité, en tant que concept criminologique changeant, revêt une signification qui va au-delà des problèmes particuliers que peuvent de temps à autre nous causer les criminels visés par cette législation. À cet égard, si nous désirons comprendre comment se construit la dangerosité elle-même de nos jours et la signification de ceci, il nous apparaît important de nous tourner d'abord vers les origines du concept, pour ensuite examiner et expliquer les changements subséquents.

\section{Dangerosité : les débuts}

Le concept de dangerosité serait apparu lors du Congrès de l'Union Internationale du Droit Pénal de 1890, à Saint-Pétersbourg, au cours de discussions sur l'introduction dans la pénalité moderne de mesures spéciales d'emprisonnement pour les individus jugés dangereux ou à risque (Ancel,1965). Le débat visait l'emprisonnement indéfini pour mettre fin au tort particulier ou au « danger » que multirécidivistes et criminels professionnels constituaient. Ce qui semblait alors les distin-

1. Une version plus longue de cet article a déjà été publiée en 1995 sous le titre : "Dangerousness, Risk and Technologies of Power" Australian and New Zealand Journal of Criminology 28: 32. 
guer du reste de la population criminelle était le fait qu'ils continuent à enfreindre la loi : « la véritable infraction est la persistance dans l'habitude délibérément acquise du crime » (Rapport du Departmental Committee on Prisons, 1895 : 303). De cette façon, ils s'excluaient eux-mêmes des deux modes de classification criminologique alors disponibles. Selon le premier mode, l'accusé était considéré comme légalement sain et donc pleinement responsable de ses actes; la sentence à imposer devait être proportionnelle au tort infligé, tout en exerçant un effet dissuasif, en accord avec les principes dominants de la pénologie victorienne : classicisme et moindre éligibilité (Pratt, 1992). Dans le second mode, l'accusé était jugé dément et donc irresponsable de ses actes. Si tel était le cas, l'individu était alors retiré des griffes de la loi pour aboutir dans celles de la psychiatrie et de l'espace restreint au sein de la justice criminelle qu' elle avait réussi à coloniser pour elle-même (un espace habituellement réservé aux meurtriers - voir Foucault, 1988 ; Wiener, 1990). Mais à cette époque, il n'existait aucune catégorie résiduelle pour les criminels dangereux ; ils étaient au-dessus de la loi qu'ils continuaient à enfreindre, et au-dessus de la psychiatrie, n'étant ni des fous de façon démontrable, ni des meurtriers. De même, l'engagement de ces groupes de récidivistes dans le crime témoignait de leur incorrigibilité, ce qui les faisait paraitre encore plus dangereux.

Toutefois, si jusqu'alors ces criminels dangereux semblaient s'être dégagés du système de justice criminelle en vigueur avec ses contraintes et ses terreurs, s'ils s'étaient eux-mêmes placés sur quelque terra nullius éphémère, hors de portée de la loi et de la psychiatrie, la pénologie moderne au tournant du $\mathrm{Xx}^{\mathrm{e}}$ siècle (Pasquino, 1991) avait colonisé cet espace et placé ses occupants sous les pouvoirs de régulation qu'elle avait introduits dans les systèmes pénaux occidentaux. La nouvelle pénologie rendait possible la conceptualisation de modes de contrôle allant bien au-delà de l'épistémè classique. Ces modes pouvaient être indéterminés de nature, sans consigner aux asiles pour fous et esprits dérangés les individus ainsi sanctionnés ; être jugé dangereux n'équivalait pas nécessairement à être jugé malade. Ainsi, parmi les contributions à la pensée pénale moderne de l'époque, mentionnons Ferri (1906) et sa négation du libre arbitre et du choix rationnel; Garofalo (1914) et le besoin de protéger la société contre les dépravations des criminels dangereux plutôt que de permettre aux garanties juridiques de les protéger ; Saleilles (1911) et la nécessité d'individualiser la peine, en se basant sur la personnalité de l'accusé plutôt que sur l'acte commis. Dans 
cet esprit, la Conférence de l'Union Internationale du Droit Pénal de 1910 (Alper et Boren, 1972) adopta finalement l'idée de la détention indéfinie pour les délinquants dangereux, une idée qui prenait forme de loi dans les sociétés modernes (Morris 1951, Pratt, 1998).

Toutes ces lois partaient de l'hypothèse que les individus encourant ces mesures spéciales démontraient des habitudes délinquantes, ce qui était à l'époque la façon de calibrer la dangerosité. Ainsi, dans le Prevention of Crime Act de 1908, qui introduisit en Angleterre la condamnation à une détention préventive, le délinquant devait avoir accumulé trois condamnations antérieures depuis l'âge de 16 ans, et on devait prouver qu'il «avait mené de façon persistante une existence malhonnête ou criminelle ». De même, le code criminel de l'Australie de l'Ouest de 1913 définissait comme criminel d'habitude toute personne de " 18 ans et plus condamnée pour un acte criminel, non punissable par la mort et ayant auparavant été condamnée à au moins deux reprises ». À ce point, cette terra nullius éphémère qu'était le concept de dangerosité avait été colonisée par des dispositions pénales modernes, capables de redessiner les limites de la classification et de la sanction autour de ses petits groupes de citoyens malhonnêtes et misérables.

\section{Dangerosité : la renaissance ?}

Entre 1920 et 1970, la législation sur les délinquants dangereux présentait les caractéristiques suivantes :

(i) la très faible utilisation des lois sur la dangerosité

Malgré les inquiétudes qui avaient contribué à l'émergence de ces lois, elles furent rarement utilisées. Citons l'exemple de la Nouvelle-Zélande où, jusqu'en 1945, on ne répertoriait que 605 individus déclarés criminels d'habitude en 40 ans d'existence de cette législation. Aux États-Unis, bien qu'initialement lié au problème de la récidive principalement chez les criminels professionnels des années 1920 (Kramer, 1982), le concept de la dangerosité a changé d'objet entre les années 1930 et 1950 pour inclure les psychopathes sexuels. Même là, Sutherland (1950 : 553) déclarait que « bien que ces lois sur la psychopathie sexuelle soient en principe dangereuses, dans la pratique elles se révèlent de peu d'importance. Elles ne sont jamais utilisées dans certains États, et rarement dans les autres... ». En Angleterre et au Pays de Galles, «de 1922 à 1928, seulement 31 criminels, en moyenne, furent condamnés à la détention préventive 
chaque année » (Radzinowitz et Hood, 1980 : 1377) ; « entre 1928 et 1945 , on ne compte que 325 incarcérations du genre (seulement 7 pour des crimes de violence) » (Morris, $1951: 65$ ) ; enfin en 1962, ce nombre avait périclité à 7 pour l'année » (Radzinowitz et Hood, 1980 : 1383).

La situation semble avoir été la même en Europe de l'Ouest, bien qu'on ait pu penser que la présence de systèmes de justice plus inquisitoriaux aurait favorisé le recours à ces mesures, puisque l'on pouvait s'attendre à une opposition moindre de la part de la magistrature de ces pays. Et en effet, dans l'ensemble de la société occidentale il semble que les trois seules juridictions à avoir exercé un usage significatif du pouvoir de condamner à la détention indéfinie pour raison de danger pour la société aient été la Russie soviétique, l'Italie fasciste et l'Allemagne nazie (Morris, 1951).

(ii) la réticence des législatures des sociétés démocratiques à abandonner les pouvoirs de la détention indéfinie qu'elles avaient accordés aux tribunaux

Même si on avait rarement recours à ces lois, les gouvernements n'en démontraient pas moins une réticence marquée à abandonner de tels pouvoirs (pour l'Angleterre, voir le Report of the Departmental Committee on Persistent Offenders, 1932). Telle était la situation malgré le poids croissant de la recherche dans l'après-guerre indiquant que lorsque ces mesures étaient utilisées, c'était généralement des criminels non dangereux qui se retrouvaient dans leurs filets (voir Advisory Council on the Treatment of Offenders, 1963 : 8). Néanmoins, le rapport de 1932 recommandait le maintien de la législation sur la dangerosité ; le Criminal Justice Bill de 1938 et le Criminal Justice Act de 1948 étendirent le principe d'indétermination en introduisant la sentence de formation corrective pour les criminels concernés, âgés entre 21 ans et 30 ans. Bien qu'abolissant la condamnation à la détention préventive, le Criminal Justice Act de 1967 n'avait fait que la remplacer en introduisant la sentence prolongée qui contenait des pouvoirs spéciaux de détention concernant les délinquants dangereux.

Il en alla de même en Nouvelle-Zélande : le Criminal Justice Act de 1954 remplaçait la déclaration de criminel d'habitude par la condamnation à la détention préventive, laquelle était à son tour fort peu utilisée (Webb, 1981) ; malgré tout, cette condamnation resta inscrite dans le Criminal Justice Act de 1967, le ministre de la Justice déclarant que « les autorités devraient avoir le pouvoir de garder les criminels [concernés] 
hors de circulation pour très longtemps " (NZPD vol. 353, p. 3629, 1967). Même aux États-Unis, la faible utilisation des lois de 1950 sur les psychopathes sexuels ne parvint pas à empêcher l'inclusion d'une clause relative aux " criminels dangereux » dans le code pénal modèle de 1962 ; dans le même esprit, le Conseil national sur le crime et la délinquance maintenait, en 1967, que « l'emprisonnement est nécessaire pour les criminels qui représentent, s'ils ne sont pas emprisonnés, un grave danger pour la société ».

\section{(iii) modifications dans la construction de la dangerosité et son mode de calcul}

De son association initiale avec les criminels habituels et professionnels, la caractérisation de la dangerosité va et vient selon les valeurs changeantes de la société, démontrant comment ce concept est une construction sociale plutôt qu'une entité ontologique. En Angleterre, la dangerosité en était venue à inclure « certains criminels sexuels [...] particulièrement ceux qui commettent des délits répétés contre les enfants et les jeunes personnes, et ceux qui corrompent les garçons » (Report of the Departmental Committee on Persistent Offenders, 1932). Au milieu du XX $\mathrm{XX}^{\mathrm{e}}$ siècle, aux États-Unis, la dangerosité adopta la forme du psychopathe sexuel (Sutherland, 1950). Dans la Grande-Bretagne de l'après-guerre, le concept régresse pour inclure « le criminel relativement banal et sans intérêt. Ce type de criminel représente le principal problème de persistance dans le crime » (Advisory Council on the Treatment of Offenders, $1963: 11$ ).

En plus d'assister à des changements de vision pour déterminer qui est considéré comme dangereux, on voit poindre des changements dans le calibrage de la dangerosité. On ne calcule plus la dangerosité uniquement sur la base des infractions antérieures d'un individu, mais en fonction de la nécessité de protéger la population contre des criminels du genre. Auparavant, seule la loi anglaise de 1908 sur la prévention du crime justifiait ces mesures de droit pénal sur la base de la "protection de la population ». Malgré des engagements fermes en Europe touchant la défense sociale au cours de la première moitié $\mathrm{du} \mathrm{XX}^{\mathrm{e}}$ siècle (voir Ancel, 1965), dans les juridictions de common law anglaise, il y avait eu beaucoup plus de circonspection face à la croissance du pouvoir de l'État sur l'individu qui semblait découler de ce concept. Après la guerre, toutefois, ce concept semble obtenir un appui beaucoup plus important de ces juridictions, qui s'étaient auparavant montrées résistantes ou tièdes. Dans la section 21(2) du Criminal Justice Act anglais de 1948, la 
dangerosité reste liée au nombre et à la régularité des infractions. Mais ce qui a changé, c'est que désormais les motifs de la sentence sont plus étroitement liés à la défense de la société (« si la cour est satisfaite qu'il est opportun pour la protection de la population que l'individu soit maintenu en captivité pour une période substantielle » (s. 21(b) — les italiques sont de moi).

C'est ainsi que Grunhut (1948: 311) soutenait que « les faits justifient la demande pour une plus forte protection de la société contre les délinquants dangereux persistants » [i.e. "les criminels d'habitude à proprement dit et les criminels professionnels »]. Le ministère de la Justice de la Nouvelle-Zélande (1954:6) déclarait que « les criminels endurcis devraient être emprisonnés pendant une longue période pour protéger la communauté, et pour qu'eux-mêmes en viennent à réaliser la futilité de leurs activités criminelles ». Le Criminal Justice Act anglais de 1967 prévoyait l'imposition de la sentence prolongée « tenant compte des antécédents du criminel et de la nécessité de protéger la population »; cette sentence ne devait être utilisée que contre « ceux qui représentaient une véritable menace pour la société ».

À partir des années 1970, je ne crois pas que l'on puisse vraiment dire qu'on ait laissé la législation sur la dangerosité tomber en désuétude (Bottoms, 1977). Jamais elle ne s'était vraiment présentée comme une option de condamnation significative, puisque de toute façon ces criminels pouvaient être condamnés à de longues périodes d'emprisonnement en vertu de mesures pénales existantes. Mais, plus symboliquement, n'était-ce pas que l'on n'avait jamais prévu que ces lois elles-mêmes puissent constituer une option véritable? Toute sur-utilisation de ces lois allait provoquer immédiatement des échos de totalitarisme - très certainement dans la période de l'après-guerre. Tel avait été le cas dans les sociétés où les droits et les intérêts des individus pouvaient être si dramatiquement minés alors que ceux de l'État se trouvaient maximisés. Par exemple, le code pénal italien de 1930 conférait au tribunal le pouvoir de déclarer certaines personnes comme ayant une tendenza a delinquere et de leur appliquer des mesures spéciales de ségrégation, bien qu'auparavant elles n'aient jamais été reconnues coupables de crime (Ancel, 1965). De la même façon, la gestapo nazie avait le pouvoir de garder en détention de protection les criminels d'habitude et les «personnes asociales » (Wolff, $1993: 5$ ).

C'est ainsi que dans l'après-guerre, alors que les démocraties commençaient à légiférer pour assurer la protection de la population, on posa des limites quant à la portée de ces mesures. De toute évidence, la 
législation sur la dangerosité de l'époque avait marqué le rôle fortement accru de l'État-providence qu'on voyait émerger à partir de 1945. De plus, le changement dans le calcul de la dangerosité, passant de la quantification des antécédents criminels à la protection de la population, était aussi un signe d'une plus grande érosion des principes de la justice classique vers un modèle commandé plutôt par la volonté de l'exécutif. Mais en même temps, les pouvoirs d'emprisonnement indéfini étaient résiduels, comme si la dangerosité représentait une sorte d'arrière-pays entre démocraties et sociétés totalitaires. Surpeupler cet arrière-pays viendrait estomper les frontières entre ces deux types de société que cette législation, avec son quasi-abandon des règles de droit, avait contribué à établir.

Si l'on examine maintenant la période depuis les années 1970, on s'apercevra que le plus étonnant peut-être dans le débat sur la dangerosité à partir de ce moment touche les changements que la classification et l'évaluation subissent à nouveau. Ce que l'on considère comme une conduite dangereuse s'applique beaucoup plus fortement aux délits violents/sexuels (répétés), le prédateur sexuel des années 1990 suivant les traces du psychopathe sexuel du milieu du $\mathrm{XX}^{\mathrm{e}}$ siècle. Mais si on assiste à la reconstitution du criminel dangereux dans la période suivant les années 1970, on constate également que le mode d'évaluation de la dangerosité devient plus raffiné. Initialement, le calcul de la dangerosité répondait aux principes du droit pénal classique : plus le nombre de crimes commis est grand, plus la sentence est sévère, jusqu'à ce que finalement les frontières du classicisme même soient ébréchées. Nous avons déjà vu comment, après la guerre, la " protection de la population » s'est insérée en tant que critère alors que l'État moderne continuait à accroître ses devoirs et responsabilités. Cependant, autour des années 1970 et par la suite, on découvre un intérêt croissant pour le genre de crime qu'un individu pourrait commettre dans le futur, plutôt que pour le nombre de crimes commis antérieurement, comme c'était le cas à l'origine (voir, par exemple, Scottish Council on Crime, 1975).

En 1977, Bottoms fit allusion de façon quasiment prophétique à cette réactivation des lois sur la dangerosité qui allait survenir par la suite, et cela comme une sorte de renaissance du concept : les gouvernements, en misant sur ce point, ont aidé à soutenir une conscience collective qui s'effilochait, conséquence des crises économiques et des changements sociaux. Mais en rester là élude deux questions : pourquoi le concept de dangerosité a-t-il pris ce virage à ce moment précis ; 
qu'est-ce qui a également rendu possible l'évaluation de la dangerosité sur la base d'une propension à commettre de futurs crimes ?

\section{Danger et modernité}

Durant les années 1940, la "protection de la population » s'imposa dans l'agenda législatif sur la dangerosité ; cette situation n'était en fait qu'un prolongement plutôt tardif des gestes posés relativement à la protection contre les risques et dangers de différentes sortes que les gouvernements occidentaux, à partir des années 1870, s'étaient préparés à offrir à leurs citoyens en guise d'assurance sociale : protection contre la maladie par le développement de services de santé ; protection contre la pauvreté lors de la vieillesse par l'octroi d'une pension ; protection contre les conséquences du chômage par le financement de «l'assurance chômage », et ainsi de suite. Toutes ces mesures représentaient une réponse collectivisée aux risques sociaux qui pouvaient s'abattre sur les individus pendant le cours normal de leur existence ; le prolongement de la protection pénale contre les criminels dangereux sortant de l'ordinaire représentait des responsabilités supplémentaires face à la sécurité des personnes que l'État-providence moderne était maintenant prêt à assumer.

Parmi les conséquences logiques de pareilles initiatives, on assista à la croissance d'une structure administrative complexe, chargée de régler et de distribuer ce « régime continu d'assistance » (Castel, 1991). Ce régime devint la responsabilité d'un spécialiste du bien-être, nouveau type de juge intéressé non pas tant par les lois et leur interprétation, que par les codes, les normes et la maximalisation du potentiel humain, qu'il s'agisse de santé, de vieillissement, de bourses d'études, de bonne conduite et ainsi de suite. Pour régler tout écart par rapport à ces normes, on établit des stratégies de discipline et de surveillance (Foucault, 1977) : discipline dans le sens de transformer l'âme de criminels particuliers, pour en faire des sujets normaux, utiles à la société et productifs grâce à du counselling et à des techniques de réhabilitation; et surveillance, destinée à des populations dans leur ensemble, par la tenue de registres, études et divers modes de classification et de distribution. De cette façon, le pouvoir de juger les individus (qui, jusqu'ici, était une question de culpabilité et d'innocence réservée aux tribunaux) se retrouva lié à d'autres pouvoirs tels que ceux de classification, d'évaluation, de diagnostic. Et on en vint à exercer le pouvoir de juger dans nombre de lieux, notamment dans les conférences 
de cas ou dans les bureaux des psychiatres, abolissant ainsi l'exclusivité des tribunaux. Par conséquent, en ce qui concerne l'administration de la justice criminelle, à partir du début du $\mathrm{XX}^{\mathrm{e}}$ siècle, nous voyons que la prise de décision par des administrateurs remplace le formalisme légal de l'École classique. Par exemple, la remise en liberté de délinquants dangereux n'est plus du ressort des juges (du moins pas dans cette fonction) mais des tribunaux et des commissions de libérations conditionnelles.

Cette protection accrue que les gouvernements étaient disposés à offrir aux citoyens présente un effet double : (i) elle maximisait le potentiel des populations en général et (ii) elle constituait une façon de réglementer les individus en assurant leur conformité aux normes ainsi établies (Foucault, 1979). On créait de cette manière une relation de dépendance entre les citoyens et leurs gouvernements. En retour d'une conformité à une gamme de normes, les gouvernements assuraient une protection contre les dangers et les risques. On donnait une série d'assurances et on créait une série d'attentes, par-dessus tout, peut-être, le «droit à la vie » dans les sociétés occidentales modernes, un droit qui démarquait ainsi la modernité autant de la période antérieure au siècle des Lumières que des autres sociétés non occidentales d'aujourd'hui.

On ne peut toutefois pas parler de tendance uniforme. Comme nous l'avons vu, la protection contre les périls du vieillissement, la maladie et ainsi de suite, avait fait des progrès notables bien avant que la « protection de la population » ne devienne un critère dans l'élaboration des lois contre les délinquants dangereux. Depuis lors, la menace au bien-être physique d'une personne a graduellement supplanté la menace à la propriété que constituaient les criminels dangereux. Et en effet, dans la période qui suit les années 1970, en même temps que les changements sociaux et économiques conçus pour maximiser le potentiel d'épanouissement (« sortir l'État de nos vies» et ainsi de suite), on voit progressivement grandir une sensibilité à la violence personnelle et aux agressions sexuelles. Le mouvement féministe, les maisons d'hébergement pour femmes, et diverses formes de prises de conscience ont contribué à intensifier la sensibilité contre ces crimes au point de rendre intolérables (par opposition à simplement criminels) le danger et la menace qu'ils posent contre la vie humaine.

Toutefois, pour poursuivre dans la pensée de Foucault, il semblerait qu'au cours de la dernière période, l'investissement dans le droit à la vie a en même temps contribué à élever les sensibilités contre des formes de comportement qui pourraient la menacer. Comme la vie en soi est 
devenue quelque chose à chérir et à protéger, les dangers qui la guettent semblent plus abondants et plus menaçants. Si nous donnons plus d'importance à la vie, inévitablement nous donnons plus d'importance aux dangers contre la vie. Comment en sommes-nous arrivés là ? En attribuant une telle valeur à la vie, on a immédiatement assisté au déclin de la sécurité, comme si la vie elle-même n'avait jamais été aussi précieuse ni aussi menacée. La dépendance accrue envers la protection par l'État à l'ère moderne a remplacé la relation plus intime et plus personnalisée de confiance et de dépendance qui existait avant la révolution industrielle (Giddens, 1990 : 113).

Ce déclin de la communauté locale remplacée par des systèmes de protection et d'ordre plus abstraits amena inévitablement une beaucoup plus grande privatisation de la vie quotidienne. En ce qui concerne notre compréhension d'un phénomène particulier comme le crime, cette situation a entrainé une dépendance envers des sources plus éloignées de nouvelles et d'information, une dépendance envers les médias plutôt qu'envers les voisins, par exemple. Dans ce cadre, la présentation des risques et des menaces provenant du phénomène du crime devient à la fois plus globalisée et plus localisée (idem). Le meurtre sexuel d'un enfant en Angleterre (comme le cas de la petite Sarah Peyne, âgée de huit ans) se retrouve immédiatement à la une dans le monde entier tout en devenant une possibilité locale, particulièrement dans des sociétés semblables, sous plusieurs rapports, à la Grande-Bretagne : si ça peut arriver là-bas, ça peut arriver ici. Chacun le sait, l'information que nous recevons sur le crime nous le fait voir " partout » tout en localisant, en même temps, la menace particulière qu'il présente pour nous, même si l'on sait combien la véritable menace de crime est inégalement répartie dans la population, et combien la menace de certains crimes particuliers varie énormément. Quoi qu'il en soit, comme nos sources d'information sur le crime mettent en lumière l'existence de "criminels dangereux », ces derniers nous apparaissent comme une menace potentielle pour chacun de nous, aussi indirecte et grande que soit la distance entre « eux » et «nous ». De cette façon, c'est comme si « une anxiété chronique et sporadiquement aiguë relativement au crime " (Sparks 1992 : 12) était devenue une caractéristique institutionnalisée de la société moderne ; le droit à la vie est accompagné de l'imminence d'une menace personnelle. 


\section{Gestion du risque et assurance}

On pourrait considérer comme une manifestation de ce phénomène la croissance et l'intensification de la législation sur la dangerosité au cours des deux dernières décennies. Toutefois, alors que ces mesures ont été introduites, la responsabilité pour la gestion des risques reliés au crime est en train de subir des changements drastiques. Les gouvernements ont commencé à décharger sur le secteur privé et les organismes bénévoles nombre des responsabilités d'assurance qu'ils assumaient depuis une centaine d'années. Le raisonnement économique et les impératifs fiscaux associés à ces changements sont assez évidents (voir Gamble, 1988) ; mais ces changements ont aussi été justifiés par la perte de confiance dans ce qui constituait alors les stratégies traditionnelles de gestion de la vie (cf. Giddens, 1990) : relativement au crime, l'écroulement de la confiance en la capacité des institutions de maintien de l'ordre et de bien-être à protéger les citoyens et à leur assurer la sécurité qui était leur tâche (Garland, 1996). Et ceci malgré l'énorme investissement de ressources publiques en spécialistes de toutes sortes dont la tâche aurait dû être de protéger les citoyens du risque de crime. La police n'est plus en mesure d'assumer seule la gestion de ce problème, clame-t-on (la police elle-même souligne le problème) ; on soupçonne les commissions de libérations conditionnelles de laisser sortir trop tôt de prison des criminels à risques élevés ; on pense que les agents de probation écrivent des rapports trop compatissants, représentant le criminel comme une victime ; les juges sont trop indulgents, et ainsi de suite. Cet écroulement de la confiance a eu un impact considérable sur la gestion du risque de crime. Deux tendances distinctes sont apparues.

D'une part, c'est comme si les gouvernements offraient à leurs citoyens, par le biais de la législation sur la dangerosité, une forme d'assurance améliorée contre les risques que représentent de tels criminels aujourd'hui. Le risque émanant de ces délinquants est considéré comme intolérable, à la fois en raison de l'affront et de l'outrage que ces crimes représentent désormais pour la sensibilité de la population, et parce que la dangerosité est maintenant associée à un comportement menaçant pour la vie. Mais c'est aussi un risque contre lequel le citoyen ordinaire n'est pas en mesure de s'assurer par lui-même, parce que la menace provenant des criminels dangereux est trop imprévisible, trop aléatoire, et que ses conséquences sont trop graves pour que les citoyens puissent s'en protéger de la manière usuelle. Et ce risque a des effets autant généralisés que localisés : la nature de la dangerosité est telle que, bien qu'il s'agisse (heureusement) d'un 
risque qui ne touche pratiquement personne, il constitue un danger qui pourrait toucher n'importe qui. Historiquement, on n'a jamais considéré que ce construit légal de dangerosité pouvait être l'apanage de personnes visiblement aliénées, de gens que l'on pouvait identifier et donc éviter à tout prix. Tel est encore le cas dans la période qui suit les années 1970 : « qu'est-ce qu'une personne dangereuse ? Il n'existe aucune entité de la sorte dans la nosologie de la psychiatrie» (Kozol et al 1972: 379). Comme conséquence, ce risque reste d'autant plus imprévisible que le danger peut provenir de gens par ailleurs ordinaires qui continuent de faire fi de la loi jusqu'à ce qu'ils soient découverts.

D'autre part, de plus en plus l'administration centrale remet la responsabilité de la gestion du risque entre les mains d'une administration locale, limitant effectivement la forme et la somme d'assurance que les gouvernements procurent. Par cela, nous n'insinuons pas que les gouvernements se retirent simplement de toute responsabilité face à la gestion du risque, mais plutôt qu'il revient aux citoyens de décider de la portée et de la nature de la couverture d'assurance. C'est aux citoyens qu'incombe de plus en plus de mobiliser leurs propres ressources pour parer aux risques qui les guettent. Cette mobilisation peut se présenter sous la forme de plans d'assurance personnels souscrits par les citoyens, de recours à des agences privées de surveillance; ou encore, les citoyens peuvent investir dans l'architecture d'espace défendable, se joindre à des groupes de protection de la communauté, comme « la surveillance de quartier », ou même s'intégrer à des regroupements de citoyens qui exercent une patrouille active du voisinage. Mais ici, la réorganisation de la gestion du risque implique la formation de nouvelles structures pour la prévention du crime, à la lumière de l'échec perçu ou de l'impuissance des méthodes plus traditionnelles de police et d'application de la loi. Le défaut d'assumer les mesures nécessaires d'autoprotection, particulièrement pour ces femmes qui deviennent victimes d'agressions sexuelles, pourrait rendre invalide toute assurance que les gouvernements, par de nouvelles lois contre les délinquants dangereux, acceptent de fournir en la matière (Stanko, 1990).

On pourrait croire en somme que les gouvernements semblent offrir plus de protection d'assurance, avec l'augmentation et le ciblage plus spécifique des législations sur la dangerosité, alors qu'en fait ils en offrent moins dans l'ensemble, conséquence de la délégation de la gestion d'un risque de crime beaucoup plus probable. De plus, ces assurances fournies par les gouvernements à l'effet qu'ils vont accroître la protection contre les individus considérés comme dangereux pourraient 
bien servir à soutenir la conscience collective, tout comme les nouveaux projets communautaires de prévention du crime : ils pourraient générer une forme de solidarité sociale entre les groupes sociaux et les individus, plutôt qu'entre les citoyens et le gouvernement comme ce fut le cas jusqu'ici. Mais plus encore, ces deux thèmes, soit la dangerosité et la prévention du crime, seraient plus représentatifs d'une réorganisation plus générale de la relation entre les gouvernements et les citoyens. Ce n'est pas tant qu'ils soutiennent une conscience collective menacée que le fait qu'ils constituent de nouvelles formes de gestion du risque dans l'ordre social qui émerge après les années 1970.

\section{Nouvelles technologies de pouvoir}

Si ce qui précède tentait d'expliquer la signification et la composition particulière de la dangerosité à l'heure actuelle, tournons-nous maintenant vers la question de son évaluation : qu'est-ce qui rend aujourd'hui possible l'évaluation de la dangerosité sur la base d'une propension à commettre de futurs délits? L'effondrement de la confiance dans les spécialistes de la justice criminelle survenue durant les années 1970 s'étendit également aux psychologues cliniciens et à leurs pouvoirs de diagnostic et d'évaluation (Steadman et Cocozza, 1974 ; Bottoms, 1977). Une nouvelle technologie de pouvoir est maintenant en train de combler le vide de prédiction ainsi créé : les méthodes actuarielles, qui consistent à appliquer des taux de base et des méthodes statistiques à la tâche de catégoriser des individus en les repérant dans des sous-groupes taxonomiques. Déjà présentes depuis plus d'un siècle dans plusieurs aspects de la société moderne, ces pratiques s'expriment dans les discours qui voient « le remplacement d'une description morale ou clinique de l'individu par un langage actuariel de calculs probabilistes et de distributions statistiques appliqués aux populations » (Feeley et Simon $1992: 452$ ).

La première étude importante de prédiction criminologique semble avoir été l'étude réalisée par Burgess et al. (1928) sur la probabilité du succès ou de l'échec des cas de libération conditionnelle : plus les scores des individus quant à des variables indépendantes considérées comme importantes pour déterminer la réussite de la libération conditionnelle étaient élevés, meilleur était leur pronostic après la remise en liberté. Glueck et Glueck (1930) ont ensuite utilisé des méthodes actuarielles pour prédire la délinquance chez des jeunes hommes sortant de maisons de correction, mais cette fois en évaluant le poids des variables indépendantes 
plutôt qu'en leur accordant simplement un score égal. En 1955, Mannheim et Wilkins produisaient les premières études importantes d'aprèsguerre sur la prédiction, utilisant alors des statistiques multivariées, leur objet d'étude étant la probabilité de réussite ou d'échec des jeunes sortant des «Borstals ». Meehl (1954:15-16) soutenait que les méthodes de prédiction actuarielles étaient à la fois plus précises et plus efficaces que l'approche clinique, « [ces résultats étant] obtenus par l'application directe d'une équation ou d'une table aux données. Ce mot ne doit pas être pris dans son sens habituellement péjoratif. Cette table, et j’insiste, ne doit pas nécessairement être une table d'individus. Cette table peut être formée d'événements ou de situations de la vie d'une personne. La propriété déterminante réside dans le fait qu'aucun jugement, qu'aucune déduction ni pondération n'est établi par un clinicien qualifié. [En effet] une fois rassemblées les données de quelque source ou type que ce soit, la prédiction elle-même pouvait être confiée à un employé de bureau » (les italiques sont de moi).

À ce stade pourtant, alors que la dangerosité était reliée à la protection de la population et, finalement, à la délinquance future, on remarqua vite l'absence de méthodes de prédiction adéquates pour réaliser ces tâches. Ainsi, selon Morris (1951:29), « les tables de prédiction n'ont pas encore été construites en Angleterre. Aux États-Unis, elles ont été élaborées et testées à la lumière de l'expérience, et il est certain qu'elles constituent des instruments de pronostic plus efficaces que « l'œil magique » ou le " gros bon sens» sur lequel nous nous fions». De même, selon Hammond et Chayen (1963 : 177), « ce qu'il faut c'est de l'information concernant la probabilité qu'un homme qui commet une infraction de violence contre la personne commettra une autre infraction de même nature; qu'un homme qui agresse sexuellement des enfants posera un geste semblable une autre fois. Il est plutôt étonnant de constater combien nous avons peu de moyens d'estimer ces probabilités ». Et selon Simon (1971 : 13), « en 1962, bien peu d'États américains utilisaient vraiment les tables de prédiction. Tel semble encore être le cas. » L'absence de technologie appropriée pour accomplir ces tâches pourrait en partie expliquer cette situation, comme le déclaraient Mannheim et Wilkins (1955 : 144) en rapport avec leur propre recherche : «[...] étant donné que nous avions en tout 60 corrélations significatives, nous aurions dû calculer en tout plus de 1200 coefficients de corrélation pour être en mesure d'étudier le chevauchement de chacune de celles-ci entre elles. Quoique cette tâche en elle-même aurait été formidable et bien au-delà du budget disponible pour l'ensemble du 
projet, ces calculs n'auraient constitué que la première étape et les calculs ultérieurs auraient été pratiquement impossibles. »

Comme telles, les méthodes actuarielles avaient encore à être reliées à la dangerosité ; comme pratique de justice criminelle, elles étaient confinées à certaines prises de décision particulières d'ordre administratif, comme la classification, la remise en liberté anticipée et ainsi de suite. Jusqu'à ce point, l'évaluation de la dangerosité restait la prérogative de psychologues cliniciens et de spécialistes du genre. Ce n'est qu'après l'effondrement de la confiance en l'expertise clinique au début des années 1970 que les méthodes actuarielles ont connu un certain essor. En 1974, Cocozza et Steadman mirent au point une échelle de prédiction de la dangerosité. Avec Floud et Young (1981 : 276), on reconnaît l'importance et les implications des méthodes actuarielles : «[...] la mode actuarielle présente de grands avantages pratiques que nous reconnaissons largement. Relativement peu coûteuse, cette méthode est précise et cohérente. Elle est efficace pour sélectionner des éléments pertinents d'information et les pondérer selon leur importance. Elle permet des classifications optimales qui fournissent les plus hautes probabilités avec un minimum d'erreurs. » Dans le même ordre d'idées, Morris et Miller (1985: 3) précisent que « les moyens de prédiction actuels se révéleront les meilleurs à notre disposition pour plusieurs décennies ». Très significatif en ce qui nous concerne, Duckitt (1988 : 14), révisant le passage des méthodes cliniques aux méthodes actuarielles dans ce domaine, pouvait déclarer : «[...] il y a dix ans, le critère à prédire n'était pas le risque de violence mais le comportement dangereux. Le concept crucial était celui de 'dangerosité', qui était un véritable attribut de l'individu, et plus spécifiquement, une catégorie de diagnostic clinique... Le changement qui est survenu par la suite consistait à rejeter complètement le concept de dangerosité en faveur du concept de risque de violence. Ce dernier est un concept franchement statistique et probabiliste, et n'indique absolument aucun attribut véritable de l'individu» (les italiques sont de moi). Là où on démontrait que le diagnostic clinique était imparfait, la démarche actuarielle allait réduire la possibilité de l'erreur humaine au moyen de la comparaison statistique de profils correspondant à la «population dangereuse » dans son ensemble. Elle devait être encore plus efficace que les cliniciens (on ferait moins de diagnostics erronés, tout en étant plus libéral et plus humain) qui étaient apparus trop prompts à classifier les criminels comme dangereux. En démocratie, tout l'intérêt de cette législation et des pouvoirs 
qu'elle conférait à des groupes de professionnels dans le domaine de la justice criminelle résidait dans le fait qu'elle ne devait être utilisée qu'avec modération, comme une sorte de mesure résiduelle.

Ce changement vers la démarche actuarielle s'accompagne de la sophistication de ses méthodes : «[...] l'inquiétude découlant des régressions multiples provoqua dans les années 1960 le recours à des techniques de regroupements hiérarchiques, comme l'analyse configurelle [...] et l'analyse des vecteurs d'interaction automatique. Ces techniques visent essentiellement à classifier une population hétérogène en sous-groupes homogènes » (Farrington et Tarling, 1988 : 18-19). Et, évidamment, l'avènement des ordinateurs dans l'administration de la justice criminelle a grandement contribué à rendre cette tâche possible. En effet, le lien qui s'établit ainsi avec « l'actuarialisme » permet la poussée vers la prédiction des crimes futurs dans la législation moderne sur la dangerosité. Hassin (1980) a démontré l'efficacité de l'ordinateur par rapport au spécialiste en ce qui concerne la libération anticipée et la prédiction du crime. Armé de son ordinateur, un nouveau type de spécialiste, le consultant en justice criminelle, est maintenant en mesure de prédire les patterns criminels, les tendances en matière de détermination de la peine et ainsi de suite.

Néanmoins, tout mouvement vers une justice actuarielle reste inégal. Au tribunal, la loi et le diagnostic clinique continuent à aller de pair, aussi difficile que puisse être cette relation (Pratt, 1998). C'est du côté de la prise de décision administrative, particulièrement pour les libérations conditionnelles, que la justice actuarielle semble avoir réalisé le plus de progrès (Farrington et Tarling, 1988 : 9). Ce pouvoir de classifier et de prédire présente un nouveau mode d'expertise avec le potentiel de conférer à ses adeptes un statut et un prestige enviables, supérieurs à ceux des cliniciens, ces rivaux d'autrefois aujourd'hui fort discrédités. En effet, la capacité de prédire, et donc, semble-t-il, de prévenir les futurs crimes ne sera jamais réduite à la simple performance cléricale annoncée par Meehl. Même si cela suffisait à la faire sortir de l'ombre de la prise de décision exécutive et à lui faire prendre sa place dans les cours de justice, l'entreprise s'annonce comme une bataille entre elle et, par exemple, une magistrature qui n'a jamais été trop portée à partager le pouvoir avec les nouveaux venus. 


\section{Discipline, surveillance et prédiction}

En conclusion, tentons de résumer la signification actuelle de la législation sur la dangerosité. D'abord, en termes de ce qui est maintenant considéré comme un danger, les lois actuelles reflètent la façon par laquelle le «droit à la vie » dans les sociétés occidentales modernes a progressivement été valorisé puis mis à risque ces derniers temps. Plus on accorde de valeur à cette attente, plus elle est mise en péril par les menaces qui semblent l'entourer. Deuxièmement, en termes de gestion de la menace, ces lois forment un aspect de la reconfiguration plus générale de la gestion du risque apparue au cours de la période suivant les années 1970 , en représentant un domaine où on semble avoir accordé plus de protection. Toutefois, non seulement le risque particulier que représente le criminel dangereux est-il minime, mais la législation sur la dangerosité elle-même continue d'être, semble-t-il, une loi rarement utilisée (voir Austin et al., 1999). Comme telle, la législation a des effets plus symboliques que réels ; il s'agit non seulement d'un reflet de l'aversion de ce qui est ressenti comme la forme la plus répréhensible de comportement de tous les temps, mais elle est aussi l'image de ce que l'État est prêt à faire pour protéger ses citoyens contre de tels comportements. En fournissant des « garanties » contre ce risque, tel qu' il existe, l'État est même prêt à aller au delà des frontières qui déterminent les limites de la loi et du châtiment dans les sociétés démocratiques. Ainsi, les lois elles-mêmes continuent d'être des pouvoirs résiduels et symboliques, prévoyant explicitement l'enfermement sur la base de critères extérieurs à la loi tels que la protection de la population et la propension à commettre de futurs crimes. Étendre ces critères à l'ensemble de la population indiquerait que nous sommes passés de la démocratie à un régime totalitaire, et poserait une menace à la valeur actuellement attribuée à la vie. Cependant, en ce qui concerne les niveaux plus généraux de protection contre le crime, les gouvernements en ont fait retomber la responsabilité sur les communautés locales elles-mêmes.

Troisièmement, en termes de l'évaluation de la dangerosité, l'émergence du pouvoir actuariel ne remplace pas les stratégies de discipline et de surveillance : en effet, comme nous l'indiquions plus tôt, ce pouvoir a fait partie intégrante de la mise en ordre et de la régulation de la société moderne, conjointement à ces autres tactiques. Mais, clairement, l'importance et l'emplacement particuliers de ces trois stratégies de contrôle vont varier de temps à autre, les changements sociaux et politiques de la période d'après 1970 permettant la croissance du pouvoir actuariel dans le 
contrôle du crime. L'effondrement de la confiance en la capacité de l'expertise sociale à gérer le problème de la criminalité, l'évolution de l'informatique, la réification de la «vie», la sensibilité aux conduites pouvant la mettre en péril, convergent pour présenter le pouvoir actuariel comme la solution aux problèmes de prédiction et de gestion du crime.

Cette situation pourrait marquer un changement important dans la régulation et le contrôle du crime et de la déviance. Par contraste avec les tactiques de discipline et de surveillance, «l'actuarialisme » implique un mode différent de régulation. Sa tâche consiste à informer, à produire une connaissance que d'autres vont utiliser, plutôt que de tenter de contrôler l'esprit des criminels et de surveiller leurs allers et venues. Par rapport à la dangerosité, les méthodes actuarielles peuvent nous fournir un profil, pour que nous puissions ensuite situer l'individu par rapport à ce profil et rendre un jugement approprié. Il ne s'agit pas de changer le comportement de ces criminels, mais bien de les identifier et de les maîtriser.

Maîtriser, alors, d'un bout à l'autre du spectre ; changer le comportement non plus tant des criminels, mais celui des victimes potentielles. Et changer leur comportement non au moyen des vieilles techniques de counselling ou d'autres techniques semblables, mais en leur permettant de faire des choix au moyen de l'information et de l'identification des risques fournies par les méthodes actuarielles. En effet, le rôle du pouvoir actuariel dans l'ordre social actuel semble reposer sur un modèle de comportement humain complètement différent de ce qui existait avant les années 1970. À cette époque, les populations dépendaient des gouvernements en matière de protection, de sécurité et de régulation. À présent, les êtres humains ont été reconvertis en machines à calculer atomisées. Ils doivent faire des « choix rationnels » en ce qui concerne la gestion du crime et la réduction du risque. Ce n'est que là où le risque est le plus minime et le plus imprévisible, soit la menace provenant des délinquants dangereux, que les gouvernements sont actuellement préparés à accroître leurs responsabilités « assurantielles ».

\section{Références}

Advisory Council on the TReatment of Offenders. 1963. Preventive Detention, London : H.M.S.O.

Alper, B. et Boren, J. 1972. Crime: An International Agenda, Lexington : Lexington Books.

ANCEL, M. 1965. Social Defence, London : Routledge and Kegan Paul. 
Austin, J., Clark, J., Hardyman, C. et Henry, A. 1999. " The Impact of 'Three Strikes and You're Out'» Punishment and Society 1 (2) : 131-162.

Botтомs, A.E. 1977. "Reflections on the Renaissance of Dangerousness » Howard Journal of Penology and Crime Prevention 16 (2) : 70-96.

BuRGESS, E. 1928. " Factors Determining Success or Failure on Parole » in The Workings of the Indeterminate Sentence Law and Parole in Illinois, sous la direction de A. Bruce. Illinois : Springfield.

CASTEL, R. 1991. «From Dangerousness to Risk » in The Foucault Effect, sous la direction de G. Burchell et al. : Brighton : Harvester Press.

Department of Justice. 1906. Annual Report to Parliament, Wellington.

Department of Justice. 1954. Annual Report to Parliament, Wellington.

FARrington, D. et TARling, R. 1988. "Criminological Prediction: An Introduction » in Prediction in Criminology, sous la direction de D. Farrington et R. Tarling. Albany : State University of New York Press.

Feeley, M. et Simon, J. 1992. "The New Penology: Notes on the Emerging Strategy of Corrections and its Implications » Criminology 30 (4) : 449-474.

FERRI, E. 1906. The Positive School of Criminology, Chicago : Charles Kerr and Co.

Floud, J. et Young, W. 1981. Dangerousness and Criminal Justice, Cambridge : Cambridge University Press.

FouCAult, M. 1977. Discipline and Punish, London : Allen Lane.

Foucault, M. 1979. The History of Sexuality, Vol. 1 : An Introduction, London : Allen Lane.

Foucault, M. 1988. «The Dangerous Individual», Pp. 125-151 in Politics, Philosophy and Culture. London : Routledge.

Gamble, A. 1988. The Free Economy and the Strong State, London : Macmillan.

Garland, D. 1996. "The Limits of the Sovereign State » British Journal of Criminology 36 (3) : 445-471.

Garofalo, R. 1914. Criminology, Boston : Little Brown.

Giddens, A. 1990. The Consequences of Modernity, Cambridge : Polity Press.

GluecK, S. et GlueCK, E. 1930. 500 Criminal Careers, New York : Harvard University Press.

Grunhut, M. 1948. Penal Reform, Oxford : Clarendon Press.

Hassin, Y. 1980. "Early Release Committees for Prisoners versus the Computer » Criminology 18 (3) : 385-397.

KozOL, H. et al. 1972. «The Diagnosis and Treatment of Dangerousness » Crime and Delinquency 18 (4) : 371-392.

Kramer, R. 1982. "From "Habitual Offenders" to "Career Criminals". The Historical Construction and Development of Criminal Categories » Law and Human Behaviour 6 (3) : 273-293.

Mannheim, H. et WiLKINS, L. 1955. Prediction Methods in Relation to Borstal Training, London : H.M.S.O. 
Meenl, P. 1954. Clinical versus Statistical Prediction: A Theoretical Analysis and Review of the Evidence, Minneapolis : University of Minnesota Press.

Morris, N. 1951. The Habitual Criminal, London : Publications of the London School of Economics.

Morris, N. et Miller, M. 1985. «Predictions of Dangerousness », Pp. 1-50 in Crime and Justice: An International Review of Research 6, sous la direction de M. Tonry et N. Morris.

PASQUiNO, P. 1991. «Criminology: The Birth of a Special Knowledge » in The Foucault Effect. Studies in Governmentality, sous la direction de G. Burchell et al. Brighton : Harvester Press.

Radzinowitz, L. et Hood, R. 1985. A History of English Criminal Law, vol. 5, London : Butterworths.

1932. Report of the Departmental Committee on Persistent Offenders. London : HMSO Cmnd 4090.

1895. Report from the Departmental Committee on Prisons. Cmnd 7702, 56 Parliamentary Papers.

Pratt, J. 1992. Punishment in a Perfect Society, Wellington : Victoria University Press.

PRATT, J. 1998. Governing the Dangerous, Sydney : Federation Press.

SAleIlles, R. 1911. The Individualisation of Punishment, Boston : Little Brown and Co.

SCOTTISH COUNCIL ON CRIME. 1975. Crime and the Prevention of Crime, London : H.M.S.O.

SimON, F. 1971. Prediction Methods in Criminology, London : H.M.S.O.

SPARKS, R. 1992. Television and the Drama of Crime, Milton Keynes: Open University Press.

Stanko, E. 1990. «When Precaution is Normal: A Feminist Critique of Crime Prevention " in Feminist Perspectives in Criminology, sous la direction de L. Gelsthorpe et A. Morris. Milton Keynes : Open University.

Steadman, H. et CocozzA, J. 1974. Careers of the Criminally Insane: Excessive Social Control of Deviance, Lexington : D.C. Heath.

Sutherland, E. 1950. "The Diffusion of the Sexual Psychopath Laws » American Journal of Sociology 56 (2) : 142-148.

WebB, P. 1981. A History of Custodial and Related Penalties in New Zealand, Wellington : Government Printer.

WIENER, M. 1990. Reconstructing the Criminal, Cambridge: Cambridge University Press.

Wolff, J. 1993. Crime Policy against Juveniles and Crime Control during the NaziPeriod in Germany, paper presented at the 11th International Congress on Criminology, Budapest. 